\title{
Apremilast in the Treatment of Plaque Psoriasis: Differential Use in Psoriasis
}

\author{
Jia C Gao', Albert G Wu', Marissa N Contento', Jacqueline M Maher', Abigail Cline² \\ 'School of Medicine, New York Medical College, Valhalla, NY, USA; ${ }^{2}$ Department of Dermatology, New York Medical College, New York, NY, USA \\ Correspondence: Abigail Cline, Department of Dermatology, New York Medical College, I90I First Avenue, New York, NY, I0029, USA, Tel + I 212 423-6262, \\ Email clinea@nychhc.org
}

\begin{abstract}
Small molecule medications like apremilast are emerging as promising options for patients with psoriasis and other inflammatory conditions. Apremilast was approved by the Food and Drug Administration in 2014 for the management of both psoriasis and psoriatic arthritis. Apremilast inhibits phosphodiesterase-4, which increases the intracellular levels of cyclic AMP, thereby reducing inflammatory cytokine production. This review aims to discuss the published evidence and evaluate the differential use of apremilast in plaque psoriasis of the body and scalp, nail psoriasis, and palmoplantar psoriasis. In clinical trials, apremilast effectively reduced the severity of different dermatological manifestations of psoriasis and improved patients' quality of life. It has an acceptable safety profile and is generally well-tolerated. Oral medications like apremilast offer an alternative route of administration which can be more convenient and appropriate for some patients. Additionally, pharmacoeconomic analyses of available anti-psoriatic systemic agents favor apremilast as a cost-effective therapeutic option.

Keywords: apremilast, efficacy, phosphodiesterase inhibitor, small molecule, plaque psoriasis, safety, scalp, nail, palmoplantar, systemic, biologic, cost-efficacy, pharmacoeconomics
\end{abstract}

\section{Introduction}

Psoriasis is an immune-mediated inflammatory disease but its pathogenesis is not fully elucidated. The upregulation of interleukin (IL)-17 promotes the inflammatory response, leading to hyperproliferation of epidermal keratinocytes that is histopathogically characteristic of plaque psoriasis. One such inflammatory pathway involves cyclic adenosine monophosphate (cAMP), an intracellular second messenger protein that plays a key role in modulating inflammatory responses. ${ }^{1}$

Apremilast is an oral phosphodiesterase 4 (PDE4) inhibitor, which elevates cAMP levels to regulate the inflammatory response responsible for psoriasis. Apremilast received US Food and Drug Administration (FDA) approval in 2014 for treatment of moderate-to-severe plaque psoriasis in adults who are candidates for phototherapy or systemic therapy. It has since received approval in multiple countries, including Canada, the European Union, and Japan. However, it has not achieved widespread dermatological use and there is a relative paucity of literature on apremilast in psoriasis compared to its use in rheumatological applications. Herein, we review the evidence on apremilast and evaluate its safety, efficacy, and utility in treating moderate-to-severe plaque psoriasis of the body and scalp as well as nail psoriasis and palmoplantar psoriasis in adults patients.

\section{Methods}

A literature search of the PubMed and Google Scholar electronic database was conducted by an independent reviewer (JG) on May 17, 2021. The search strategy involved the following combination of terms and Boolean operators: (apremilast) AND (psoriasis). Filters were applied to only include clinical trials and randomized controlled trials from 2008 onwards.

Seventy-three articles were imported into an online reference screening and data extraction tool (Covidence systematic review software, Veritas Health Innovation, Melbourne, Australia. Available at www.covidence.org) to 
remove duplicates. After removal of duplicates studies, four independent reviewers (AW, JG, JM, MC) screened the titles and abstracts of the forty-four unique papers for inclusion or exclusion based on our predetermined criteria. Twenty-four papers met inclusion and exclusion criteria for full-text review and subsequent data extraction. Additional articles identified by hand search were included for review.

\section{Inclusion Criteria}

1. Original human studies with in vivo experimental design.

2. Studies on dermatologic indications for apremilast.

\section{Exclusion Criteria}

1. Literature review articles, commentaries, and abstract only papers.

2. Studies reporting on non-dermatologic indications for apremilast, such as psoriatic arthritis.

3. Articles not written in or translated to English.

\section{Apremilast: Mechanism of Action}

Apremilast is an orally administered PDE4 inhibitor whose modulatory effects have been described in many in vitro and in vivo human studies. PDE4 is a T-cell inhibitor which downregulates production of inflammatory cytokines. PDE4 is also expressed in keratinocytes, which are strongly implicated in the histopathology development of psoriasis through hyperproliferation of this structural cell type. ${ }^{1}$

PDE4 inhibition leads to increase in cAMP, thereby increasing levels of anti-inflammatory mediators like IL-10 and decreasing production of pro-inflammatory tumor necrosis factor-alpha (TNF-alpha), IL-23, and interferon-gamma (IFNgamma) (Figure 1). In in vitro studies, apremilast decreased the number of myeloid dendritic cells and T cells infiltrating the epidermal and dermal layers of psoriatic skin. ${ }^{1}$

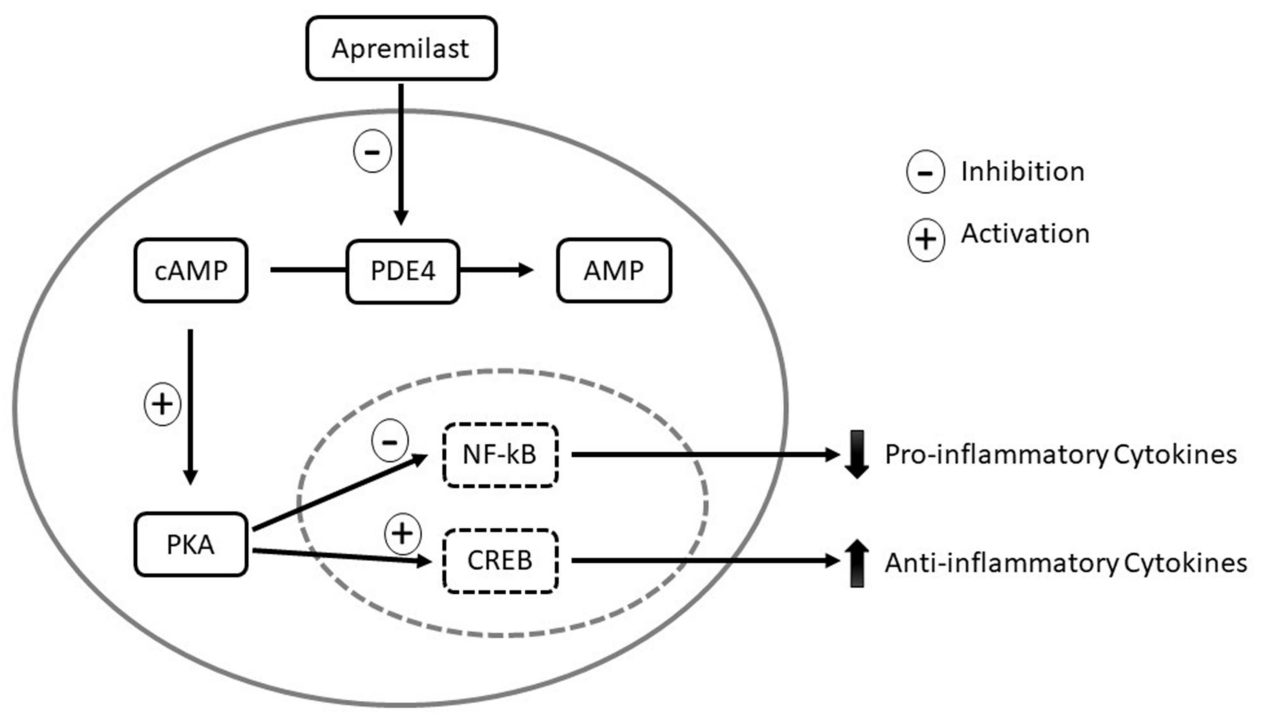

Figure I Mechanism of action of apremilast. In monocytes and dendritic cells, PDE4 degrades cAMP to AMP. When apremilast inhibits PDE4, intracellular cAMP levels increase and activate PKA. PKA activation results in phosphorylation of the transcription factors CREB and NF- $\mathrm{K} B$. Phosphorylation leads to activation of CREB, which increases anti-inflammatory cytokines, such as IL-10. Phosphorylation of NF- $\kappa B$ results in inhibition of transcriptional activity, thereby decreasing expression of proinflammatory cytokines, including IL-23, TNF- $\alpha$, and IFN- $\gamma$. The decreased production of inflammatory mediators reduces inflammation and proliferation of keratinocytes in psoriatic skin.

Abbreviations: cAMP, cyclic adenosine monophosphate; CREB, cAMP responsive element-binding protein; IFN, interferon; IL, interleukin; NF- $\mathrm{B} B$, nuclear factor-kappa B; PDE4, phosphodiesterases; PKA, protein kinase A; TNF- $\alpha$, tumor necrosis factor-alpha. 


\section{Apremilast: Pharmacodynamics and Pharmacokinetics}

Apremilast demonstrates linear pharmacokinetics. Drugs with linear pharmacokinetics are characterized by a half-life and clearance rate that are respectively independent of drug concentration and dose and scheduling. Many byproducts of metabolism have been identified but do not play a role in apremilast's pharmacological activity. Its elimination half-life is approximately 6-9 hours and average plasma clearance rate is about $10 \mathrm{~L} / \mathrm{h}^{2}$

Apremilast undergoes oxidative metabolism by cytochrome P450 enzymes, primarily CYP3A4, CYP1A2, and CYP2A6. Decreased apremilast exposure and subsequent efficacy may occur with CYP450 inducers like phenobarbital, carbamazepine, phenytoin, rifampin, and St. John's wort. However, there are no significant interactions with coadministration of ketoconazole, methotrexate, or oral contraceptive agents. ${ }^{3}$

Dosage reduction is required in patients with creatinine clearance $<30 \mathrm{~mL} / \mathrm{min}$ or an estimated glomerular filtration rate $<30$. No dosage adjustments are required for female or elderly patients aged 65 years or older, although their exposure may be slightly increased. There was no difference in pharmacokinetics among patients of different racial or ethnic background. ${ }^{4}$

\section{Differential Efficacy of Apremilast on Plaque Psoriasis: Key Evidence from Phase 3 Clinical Trials Plaque Psoriasis of the Body}

The Efficacy and Safety Trial Evaluating the Effects of Apremilast in Psoriasis (ESTEEM) 1 and ESTEEM 2, also known as PSOR-1 and PSOR-2, respectively, are two pivotal phase 3 clinical trials evaluating the safety and efficacy of apremilast for plaque psoriasis (Table 1). Across both ESTEEM 1 (NCT01194219) and ESTEEM 2 (NCT01232283) trials, a sum of 1255 patients were randomized 2:1 to apremilast $30 \mathrm{mg}$ twice daily $(\mathrm{n}=836)$ or a placebo $(\mathrm{n}=419)$. Patients were aged 18 years or older, with chronic, moderate-to-severe plaque psoriasis for at least 12 months, defined as a Psoriasis Area Severity Index (PASI) score $\geq 12$, Body Surface Area (BSA) $\geq 10 \%$, static Physician Global Assessment (sPGA) score $\geq 3$ and were eligible to receive phototherapy or systemic therapy. ${ }^{4,5}$

The trials were conducted in three stages. During Period A (Weeks 0-16), or the placebo-controlled phase, patients were randomized to receive apremilast $30 \mathrm{mg}$ twice daily or a placebo. This was followed by Period B (Weeks 16-32), or the maintenance phase, placebo patients were switched to apremilast. Finally, Period C (Weeks 32-52), or the treatment withdrawal phase, patients receiving apremilast since Week 0 and achieving PASI $\geq 75$ in ESTEEM 1 or PASI $\geq 50$ in ESTEEM 2 were re-randomized to either continue apremilast or switch to placebo. ${ }^{4,5}$

The primary endpoints of ESTEEM 1 and ESTEEM 2 were the proportion of patients achieving a 75\% reduction in their PASI score (PASI-75) by the end of the placebo-controlled period A (Week 16). In both trials, PASI-75 response was significantly higher in the apremilast treatment group compared to the placebo group: ESTEEM 1 (33.1\% of apremilast vs $5.3 \%$ of placebo; 95\% confidence interval [CI], p $<0.0001)$, ESTEEM 2 (28.8\% vs 5.8\%; $<0.001)$. The major secondary endpoints of sPGA score of 0 (clear) or 1 (almost clear) with $\geq 2$-point reduction from baseline were also achieved by significantly more patients receiving apremilast vs placebo in both studies: ESTEEM 1 (21.7\% vs 3.9\%; $\mathrm{p}<0.0001)$, ESTEEM 2 (20.4\% vs 4.4\%; $<<0.001)$ (Table 1). ${ }^{4,5}$

Table I Summary of Key Efficacy Data of Apremilast in Plaque Psoriasis: Results from Phase 3 ESTEEM Trials

\begin{tabular}{|l|c|c|c|c|}
\hline Study & Treatment Arm & Number of Patients & PASI-75 Response & sPGA Response \\
\hline ESTEEM I & Apremilast 30 mg BID & 562 & $186(33.1 \%)$ & $122(21.7 \%)$ \\
& Placebo & 282 & $15(5.3 \%)$ & $1 \mathrm{I}(3.9 \%)$ \\
& Total & 844 & $79(28.8 \%)$ & $56(20.4 \%)$ \\
ESTEEM 2 & Apremilast 30 mg BID & 274 & $16(5.8 \%)$ & $12(4.4 \%)$ \\
& Placebo & 137 & & \\
& Total & $\mathbf{4 I}$ & & \\
\hline
\end{tabular}

Abbreviation: BID, twice daily. 
In ESTEEM 1, 64 of 77 patients (83.1\%) originally receiving apremilast since the beginning of study lost PASI-75 response during the treatment withdrawal Period C (Weeks 32 to 52). The median time to first loss of PASI-75 response after re-randomization to a placebo at Week 32 was 5.1 weeks. Similarly, 32 of 62 patients (52\%) re-randomized from apremilast to placebo during Period C lost more than 50\% of their PASI-50 improvement at a median time of 12.4 weeks after re-randomization. However, in both trials, patients who lost PASI improvement during the treatment withdrawal period, the majority (ESTEEM 1: 70.3\%; ESTEEM 2: 66\%) regained PASI-75 and PASI-50 improvement, respectively, after re-initiation of apremilast therapy. ${ }^{4,5}$

Oral medications like apremilast are among a Newer class of available systemic medications for psoriasis, whereas biological agents like etanercept are more commonly used. In a phase $3 \mathrm{~b}$ study entitled Evaluation in a PlaceboControlled Study of Oral Apremilast and Etanercept in Plaque Psoriasis (LIBERATE), orally-administered apremilast $30 \mathrm{mg}$ twice daily was compared to weekly subcutaneous injections of etanercept $50 \mathrm{mg}$. Both apremilast (39.8\%) and etanercept (48.2\%) were significantly more effective at achieving PASI-75 by week 16 when compared to a placebo $(11.9 \%, \mathrm{p}<0.0001)$. Although the number of patients on etanercept achieving PASI-75 was higher than that of apremilast, the study was not powered to determine if a statistically significant difference between the groups exist. ${ }^{6}$

Overall, apremilast $30 \mathrm{mg}$ twice daily is effective at improving moderate-to-severe plaque psoriasis of the body. Although patients can lose treatment response within weeks of apremilast discontinuation, they are able to regain PASI improvement upon restarting treatment.

\section{Scalp Psoriasis}

Scalp psoriasis may encompass only a small BSA, but it presents a challenge to commonly used topical therapies due to difficulty of application in hair-bearing regions of the body. The Study of the Efficacy and Safety of Apremilast in Subjects with Moderate to Severe Plaque Psoriasis of the Scalp (STYLE, PSOR-3) was a phase 3, multicenter, randomized, double-blind, placebo-controlled study evaluating the efficacy and safety of apremilast for scalp psoriasis. ${ }^{6}$ The treatment group received apremilast $30 \mathrm{mg}$ twice daily until Week 16. At Week 16, placebo group patients were switched to apremilast through Week 32. No concomitant medications were allowed. Of the 303 patients randomized; 201 patients received apremilast and 102 received a placebo drug. From the original 303, only 252 patients completed the placebo-controlled stage of the trial through Week 16. Similar to the ESTEEM 1 and 2 trials, treatment doses were titrated in $10 \mathrm{mg}$ increments over the first week of treatment to reduce potential for gastrointestinal adverse events. ${ }^{7}$

The primary endpoint was the proportion of patients achieving Scalp PGA (ScPGA) response (score of 0 [clear] or 1 [almost clear]) with at least 2-point reduction from baseline at Week 16. Significantly more patients treated with apremilast met the primary endpoint compared to patients who received the placebo $(43.3 \%$ vs $13.7 \%$; $95 \%$ CI, $\mathrm{p}<0.0001)^{7}$

The major secondary endpoints were the proportion of patients with at least 4-point improvement from baseline in Whole Body Itch Numeric Rating Scale (NRS) and Scalp Itch NRS at Weeks 2, 4, 8, 16 and change from baseline in Dermatology Life Quality Index (DLQI) score at Week 16. At Week 16, significantly more patients receiving apremilast noticed improvement in the Whole Body NRS (45.5\% vs $22.5 \%$; $<0.0001)$, Scalp Itch NRS (47.1\% vs $21.1 \%$; $\mathrm{p}<0.0001)$, and DLQI scores $(-6.7 \mathrm{vs}-3.8 ; \mathrm{p}<0.0001)^{7}$

Similarly, in the subgroup analyses of ESTEEM 1 and ESTEEM 2 patients, significantly more apremilast patients achieved an ScPGA score of 0 or 1 than patients on the placebo by Week 16 (ESTEEM 1: 46.5\% vs 17.5\%, p<0.0001; ESTEEM 2: $40.9 \%$ vs $17.2 \%, \mathrm{p}<0.001)^{8}$

Apremilast treatment is associated with significantly better improvements in scalp psoriasis as compared to placebo. Currently, studies of other oral and systemic treatments for scalp psoriasis are limited so comparative efficacy is unknown.

\section{Nail Psoriasis}

Nail psoriasis involves an even smaller BSA, than compared to scalp or body psoriasis, but it is disproportionally associated with severely impaired quality-of-life and can be especially difficult to treat and refractory to many available 
therapies. Apremilast produces rapid and sustained improvements in DLQI and Nail Psoriasis Area and Severity Index (NAPSI) scores, as demonstrated in several clinical trials of patients taking apremilast $30 \mathrm{mg}$ twice daily. ${ }^{4,5,-11}$ In addition to investigator-based clinical outcome measures, ultrasonography-based parameters of nail health showed significant improvements and structural restoration of the nail matrix and nail bed. ${ }^{10}$

\section{Palmoplantar Psoriasis}

Palmoplantar psoriasis (PPP), also known as palmoplantar pustulosis or palmoplantar pustular psoriasis (PPPP), is another variant of psoriasis which can be severely debilitating. It affects the palms and soles and is thought to be less common than plaque psoriasis affecting the body and scalp but a pooled analysis of the phase 2b PSOR-005 and phase 3 ESTEEM 1 and ESTEEM 2 clinical trials found higher than expected prevalence of moderate-to-severe palmoplantar involvement among these study participants (10.1\%). ${ }^{12}$ However, controlled trials specifically examining the use of apremilast for PPP are scant. In a case series of six patients with severe and refractory PPP, apremilast $30 \mathrm{mg}$ twice daily led to improvements in all patients' PGA scores from their baseline of 3 or 4 to PGA scores of 1 (almost clear) or 0 (clear) after 12 weeks of use. ${ }^{13}$

\section{Patient-Reported Outcomes}

Psoriasis has a profound impact on patients' health-related quality of life (HRQOL). In addition to primary endpoint measures of efficacy, we review the patient-reported outcomes (PROs) of apremilast on plaque psoriasis. PROs include mental health status, pruritus severity, daily functioning, and work productivity, to name a few. These indicators may provide a more comprehensive view of the impact that psoriasis and its treatment can have on patients. ${ }^{14}$

PROs can be assessed with a variety of validated instruments, such as the DLQI, Patient Health Questionnaire-8 (PHQ-8), 36-Item Short-Form Health Survey version 2 (SF-36v2), European Quality of Life-5 Dimensions Questionnaire (EQ-5D), and Work Limitations Questionnaire-25 (WLQ-25). However, the minimal clinically improvement difference (MCID) in these PRO assessments varies from one instrument to the next.

Apremilast treatment was consistently associated with better PROs as compared with placebo among patients in the ESTEEM 1 and ESTEEM 2 trials. The mean changes from baseline in DLQI and SF-36v2 mental component summary exceeded the MCID threshold, suggesting that the improvements were meaningful to patients. HRQOL gains emerged by Week 4, gradually increased, and were sustained through Week 32. Further, work productivity, as measured by WLQ-25, also increased among apremilast patients, especially those who achieved PASI-75. The durability of improvements in PROs requires longer term monitoring and analyses beyond 32 weeks. ${ }^{14}$

Additionally, a Phase 4 multicenter, randomized controlled trial in patients with low BSA involvement (5-10\% BSA) known as Evaluating Apremilast in a Phase IV Trial of Efficacy and Safety in Patients with Moderate Plaque Psoriasis (UNVEIL) demonstrated significantly better patient satisfaction with the safety, efficacy, and convenience of apremilast compared with placebo. ${ }^{15}$

\section{Safety and Tolerability Profile Most Common Adverse Events}

Apremilast was generally well-tolerated with an acceptable safety profile among patients in clinical trials. A long-term, pooled safety analysis monitored 1250 patients ( 832 treated with apremilast, 418 treated with placebo) in the ESTEEM trials for more than 156 weeks for treatment-related adverse events (AEs) (Table 2). The most common reasons for discontinuing the study were lack of efficacy (34.7\%) and patient withdrawal from the study (18.5\%). Most AEs presented within the first week of dosing and self-resolved within one month. AE rates decreased over time and did not increase with longer exposure to apremilast treatment. Few patients discontinued apremilast therapy due to AEs. ${ }^{8}$

The most commonly reported adverse events (AEs) were diarrhea $(\mathrm{n}=221,18.7 \%)$, nausea $(\mathrm{n}=195,16.5 \%)$, upper respiratory tract infection $(\mathrm{n}=227,19.2 \%)$, nasopharyngitis $(\mathrm{n}=196,16.6 \%)$, and headache $(\mathrm{n}=201,17.0 \%)($ Table 2$)$. Severe and serious AEs were uncommon and comparable between treatment and placebo groups, occurring in around $2 \%$ of patients in each group in both trials. ${ }^{4,5,8}$ Secretory diarrhea, nausea, and vomiting were thought to be induced by PDE4 inhibition; a similar mechanism is implicated in caffeine-induced diarrhea. ${ }^{8}$ 
Table 2 Summary of Key Safety Data of Apremilast in Plaque Psoriasis: Results from Long-Term Pooled Safety Analysis of ESTEEM Trials

\begin{tabular}{|l|c|c|c|c|}
\hline \multirow{2}{*}{ Adverse Events } & \multicolumn{3}{|c|}{ Number of Patients Experiencing Adverse Event During Each Exposure Period } \\
\cline { 2 - 5 } & Weeks 0-52 & Weeks 52-104 & Weeks 104-156 & *Total (Weeks 0-156+) \\
\hline Diarrhea & $205(17.3 \%)$ & $15(2.3 \%)$ & $7(1.7 \%)$ & $221(18.7 \%)$ \\
Nausea & $186(15.7 \%)$ & $5(0.8 \%)$ & $6(1.5 \%)$ & $195(16.5 \%)$ \\
URTI** & $184(15.5 \%)$ & $58(8.9 \%)$ & $27(6.7 \%)$ & $227(19.2 \%)$ \\
Headache & $181(15.3 \%)$ & $43(6.6 \%)$ & $12(1.9 \%)$ & $201(17.0 \%)$ \\
\hline
\end{tabular}

Notes: *Total number of patients does not equal the sum of the three 52-week exposure periods because some of the patients are counted more than once in each of the three timeframes if they experienced the adverse event at more than one period throughout the study. **Upper respiratory tract infection.

PDE inhibition can affect other organ systems like adipose tissue, which may contribute weight loss. ${ }^{16}$ In one study, $21.9 \%$ of patients sustained weight loss $>5 \%$ of their baseline body weight. ${ }^{8}$ The median (mean) weight loss was 1.40 (2.08) $\mathrm{kg}$, with $19 \%$ of patients experiencing weight loss $>5 \%$ of their baseline body weight. However, no patients discontinued the trial due to weight loss and weight loss did not lead to any known sequelae. Importantly, there was no association between gastrointestinal AEs and weight loss. ${ }^{4}$

\section{Mental Health-Related Adverse Events}

One patient on apremilast attempted suicide during the first year of treatment and one patient receiving the placebo drug completed suicide in the ESTEEM trials. Apremilast has a warning label for risk of depression due to increased incidence of depression at Week 16 in the apremilast treatment group of the ESTEEM trials (1.3\% vs $0.4 \%)$. However, depression and increased suicidality are independently associated with psoriasis. ${ }^{8}$

\section{Serious Adverse Events}

Incidences of cardiac AEs and malignancies were similar to the general population of patients with psoriasis. Three deaths occurred in patients receiving apremilast during years 1,2, and 3 of treatment due to heart failure and stroke but they were not thought to be treatment-related AEs. ${ }^{8}$

No serious opportunistic infections occurred, and no clinical reactivation of tuberculosis (TB) was observed, despite 7 patients with a prior history of TB. ${ }^{8}$ Latent TB reactivation is a well-documented concern and adverse event that variably occurs in patients on some systemic biological agents with immunosuppressive effects. In studies of patients taking biological agents and small molecule inhibitors like apremilast, latent TB reactivation is not associated with apremilast use. $^{8,17}$

\section{Contraindications}

Currently, use of apremilast for plaque psoriasis in specific populations like pregnant women and pediatric patients is unknown. Apremilast is contraindicated in patients who have a known hypersensitivity to oral PDE inhibitors and is not indicated in pregnancy and pediatric patients under the age of 18 .

\section{Discussion}

Apremilast is an orally administered medication for moderate-to-severe plaque psoriasis. Topical corticosteroids remain the first line therapy for psoriasis and oral systemic medications are reserved for refractory cases or those involving an extensive BSA. In clinical trials, apremilast effectively reduced the severity of plaque psoriasis of the body and scalp and improved patients' quality of life. Treatment response to apremilast was maintained over the 52-week long phase 3 clinical trials and patients who experienced loss of efficacy during treatment withdrawal were able to regain response upon restarting apremilast therapy. ${ }^{4,5}$

Apremilast has an acceptable safety profile and is generally well-tolerated with short-term and long-term use. The most common side effects emerge in the early stages of initiating treatment and are typically mild and self-resolve within 
weeks. The treatment-related weight loss is common but unrelated to the other gastrointestinal side effects of nausea, vomiting, and diarrhea. Importantly, there is no increased incidence of infections or malignancies with apremilast therapy.

Apremilast carries a warning for increased risk of depression, but psoriasis is known to independently negatively affect patients' mental health and quality of life. Further, apremilast treatment improves many PRO indicators like DLQI and PHQ-8, as well as work productivity. ${ }^{14}$

Oral medications offer an alternative route of administration which can be more convenient and appropriate for some patients who have difficulty adhering to other forms of psoriasis therapy. Particularly for scalp psoriasis, oral medications are preferred by some patients to topical medications due to difficulty of application to hair-bearing regions. When psoriasis involves an extensive BSA, topical therapies can be impractical and systemic medications are considered. Injectables and oral medications are among these systemic agents but some patients may have a strong aversion to routine injections. ${ }^{18}$ Additionally, unlike some oral medications for psoriasis, there is no need for routine laboratory monitoring in patients taking apremilast. In long-term studies of safety, clinical chemistry and hematology abnormalities and shifts outside of the normal range were rare, reversible, and comparable between treatment and placebo groups. ${ }^{8}$

As with all maintenance medications for chronic diseases, the cost of treatment is an important factor for most patients and impacts the feasibility of adhering to the treatment plan. Currently, for patients who do not qualify for patient assistance programs or insurance reimbursement, the monthly out-of-pocket cost of apremilast rivals that of some biological injectable agents. ${ }^{19,20}$ As of September 2021, the apremilast patent has been upheld, delaying the introduction of a more affordable generic oral PDE4 inhibitor to the market.

The introduction and use of small molecule inhibitors like apremilast carries economic implications for healthcare systems and spending. Apremilast has the added utility of delaying the use of biological agents, some of which can be more costly and associated with more adverse effects. ${ }^{21}$ Further, cost-efficacy analyses of systemic anti-psoriatic agents favor apremilast to most other commonly-used biological agents. ${ }^{22}$

\section{Conclusion}

Apremilast is efficacious in treating plaque psoriasis, portends no increased risk of tuberculosis, requires no laboratory monitoring, is administered orally, and may lead to weight loss - a side effect which may be valuable to patients. The long-term safety and efficacy of apremilast should be further investigated as it remains to be seen whether there is reduced immunosuppression associated with apremilast compared with biologic agents.

\section{Funding}

There is no funding to report.

\section{Disclosure}

The authors report no conflicts or competing interests in this work.

\section{References}

1. Schafer P. Apremilast mechanism of action and application to psoriasis and psoriatic arthritis. Biochem Pharmacol. 2012;83(12):1583-1590. doi:10.1016/j.bcp.2012.01.001

2. Otezla [Full Prescribing Information]. Celgene Corporation, Summit, NJ. Available from: https://www.pi.amgen.com/united_states/otezla/otezla_pi_ english.pdf. Accessed November 10, 2021.

3. Deeks ED. Apremilast: a Review in Psoriasis and Psoriatic Arthritis. Drugs. 2015;75(12):1393-1403. doi:10.1007/s40265-015-0439-1

4. Papp K, Reich K, Leonardi CL, et al. Apremilast, an oral phosphodiesterase 4 (PDE4) inhibitor, in patients with moderate to severe plaque psoriasis: results of a Phase III, randomized, controlled trial (Efficacy and Safety Trial Evaluating the Effects of Apremilast in Psoriasis [ESTEEM] 1). $J \mathrm{Am}$ Acad Dermatol. 2015;73(1):37-49. doi:10.1016/j.jaad.2015.03.049

5. Paul C, Cather J, Gooderham M, et al. Efficacy and safety of apremilast, an oral phosphodiesterase 4 inhibitor, in patients with moderate-to-severe plaque psoriasis over 52 weeks: a phase III, randomized controlled trial (ESTEEM 2). Br J Dermatol. 2015;173(6):1387-1399. doi:10.1111/ bjd.14164

6. Reich K, Gooderham M, Green L, et al. The efficacy and safety of apremilast, etanercept and placebo in patients with moderate-to-severe plaque psoriasis: 52-week results from a phase IIIb, randomized, placebo-controlled trial (LIBERATE). J Eur Acad Dermatol Venereol. 2017;31 (3):507-517. doi:10.1111/jdv.14015 
7. Van Voorhees AS, Stein Gold L, Lebwohl M, et al. Efficacy and safety of apremilast in patients with moderate to severe plaque psoriasis of the scalp: results of a phase 3b, multicenter, randomized, placebo-controlled, double-blind study. J Am Acad Dermatol. $2020 ; 83(1): 96-103$. doi:10.1016/j.jaad.2020.01.072

8. Crowley J, Thaçi D, Joly P, et al. Long-term safety and tolerability of apremilast in patients with psoriasis: pooled safety analysis for $\geq 156$ weeks from 2 phase 3, randomized, controlled trials (ESTEEM 1 and 2). J Am Acad Dermatol. 2017;77(2):310-317.e311. doi:10.1016/j.jaad.2017.01.052

9. Lanna C, Cesaroni GM, Mazzilli S, et al. Apremilast as a target therapy for nail psoriasis: a real-life observational study proving its efficacy in restoring the nail unit. J Dermatolog Treat;2020. 1-5. doi:10.1080/09546634.2020.1801976

10. Muñoz-Santos C, Sola-Ortigosa J, Vidal D, Guilabert A. Apremilast improves quality of life and ultrasonography parameters in patients with nail psoriasis: a prospective cohort study. J Dermatol. 2021;48(10):1593-1596. doi:10.1111/1346-8138.16074

11. Oak ASW, Ho-Pham H, Elewski BE. Improvement of 11 patients with nail psoriasis with apremilast: results of an investigator-initiated open-label study. J Am Acad Dermatol. 2020;83(6):1830-1832. doi:10.1016/j.jaad.2020.05.087

12. Bissonnette R, Pariser DM, Wasel NR, et al. Apremilast, an oral phosphodiesterase-4 inhibitor, in the treatment of palmoplantar psoriasis: results of a pooled analysis from Phase II PSOR-005 and Phase III Efficacy and Safety Trial Evaluating the Effects of Apremilast in Psoriasis (ESTEEM) clinical trials in patients with moderate to severe psoriasis. J Am Acad Dermatol. 2016;75(1):99-105. doi:10.1016/j.jaad.2016.02.1164

13. Ständer S, Syring F, Ludwig RJ, Thaçi D. Successful Treatment of Refractory Palmoplantar Pustular Psoriasis With Apremilast: a Case Series. Front Med. 2020;7:543944. doi:10.3389/fmed.2020.543944

14. Thaçi D, Kimball A, Foley P, et al. Apremilast, an oral phosphodiesterase 4 inhibitor, improves patient-reported outcomes in the treatment of moderate to severe psoriasis: results of two phase III randomized, controlled trials. J Eur Acad Dermatol Venereol. 2017;31(3):498-506. doi:10.1111/jdv. 13918

15. Strober B, Bagel J, Lebwohl M, et al. Efficacy and Safety of Apremilast in Patients With Moderate Plaque Psoriasis With Lower BSA: week 16 Results from the UNVEIL Study. J Drugs Dermatol. 2017;16(8):801-808.

16. Zhang R, Maratos-Flier E, Flier JS. Reduced adiposity and high-fat diet-induced adipose inflammation in mice deficient for phosphodiesterase 4B. Endocrinology. 2009;150(7):3076-3082. doi:10.1210/en.2009-0108

17. Hagberg KW, Persson R, Vasilakis-Scaramozza C, et al. Herpes Zoster, Hepatitis C, and Tuberculosis Risk with Apremilast Compared to Biologics, DMARDs and Corticosteroids to Treat Psoriasis and Psoriatic Arthritis. Clin Epidemiol. 2020;12:153-161. doi:10.2147/CLEP.S239511

18. Balogh EA, Bashyam AM, Ghamrawi RI, Feldman SR. Emerging systemic drugs in the treatment of plaque psoriasis. Expert Opin Emerg Drugs. 2020;25(2):89-100. doi:10.1080/14728214.2020.1745773

19. How much does Otezla ${ }^{\circledR}$ (APREMILAST) cost?: official Website. Otezla ${ }^{\circledR}$ (apremilast) - Official Website. Availablr from: https://www.otezla.com/ plaque-psoriasis-treatment/how-much-does-otezla-cost. Accessed November 21, 2021.

20. Paying for ENBREL: the cost of ENBREL and financial support options. Enbrel ${ }^{\circledR}$ (etanercept). Availablr from: https://www.enbrel.com/financialsupport. Accessed November 21, 2021.

21. Tanaka M, Ozeki Y, Matsuyama F, Murata T, Imafuku S, Nakamura T. Apremilast Prolongs the Time to First Biologic Therapy in Japanese Patients with Psoriasis. Dermatol Ther (Heidelb). 2021;12:451-466. doi:10.1007/s13555-021-00659-w

22. Kromer C, Celis D, Sonntag D, Peitsch WK. Biologicals and small molecules in psoriasis: a systematic review of economic evaluations. PLoS One. 2018;13(1):e0189765. doi:10.1371/journal.pone.0189765

Clinical, Cosmetic and Investigational Dermatology is an international, peer-reviewed, open access, online journal that focuses on the latest clinical and experimental research in all aspects of skin disease and cosmetic interventions. This journal is indexed on CAS. The manuscript management system is completely online and includes a very quick and fair peer-review system, which is all easy to use. Visit http://www. dovepress.com/testimonials.php to read real quotes from published authors.

Submit your manuscript here: https://www.dovepress.com/clinical-cosmetic-and-investigational-dermatology-journal 